\title{
Tangence
}

\section{Donald Barthelme Fades Away from Here to Eternity}

\section{Jean-Pierre Vidal}

Numéro 36, mai 1992

La lecture littéraire

URI : https://id.erudit.org/iderudit/025716ar

DOI : https://doi.org/10.7202/025716ar

Aller au sommaire du numéro

Éditeur(s)

Tangence

ISSN

0226-9554 (imprimé)

1710-0305 (numérique)

Découvrir la revue

Citer ce document

Vidal, J.-P. (1992). Donald Barthelme Fades Away from Here to Eternity.

Tangence, (36), 105-108. https://doi.org/10.7202/025716ar d'utilisation que vous pouvez consulter en ligne.

https://apropos.erudit.org/fr/usagers/politique-dutilisation/ 


\section{DOCWMPNi}

\section{Donald Barthelme Fades Away from Here to Eternity ${ }^{1}$}

Jean-Pierre Vidal

à Bertrand Gervais

Tandis que nous attendions Don Bartolomeo, commissaire chez Sotheby's, qui devait nous exposer les raisons de la mévente récente d'un Van Goff, pourtant sublime, nous nous prîmes à songer à la difficulté des temps. Faudrait-il donc aller, devant l'indifférence manifeste et universelle à l'endroit de tout ce qui se targue d'art, jusqu'à accepter que cette forme particulière et fort ancienne d'activité humaine n'ait été que transitoire? Nous ne savions trop que penser.

Tandis que je m'avance du plus vite que je peux à travers la foule qui, inexplicablement pour cette heure de la journée, a envahi les couloirs du métro, afin de me rendre à l'Université où l'on m'attend pour parler de la dépression qui frappe aussi le milieu des collectionneurs d'art (un Van Goff, pourtant d'une beauté rare, n'a pas trouvé preneur à la vente que j'ai dirigée récemment chez Sotheby's), je ne puis m'empêcher de songer à Théo et à sa passion des modèles réduits. Théo, mon frère bien aimé.

Qu'on le veuille ou non, l'attachement qu'éprouvent certains pour des activités dont l'effet social est maintenant à peu près

1 Écrit en regard de * Engineer-private Paul Klee misplaces an Aircraft between Milbertshofen and Cambrai, March 1916*, de Donald Barthelme (Forty stories, Penguin books, 1987) et avec quelques phrases de Vincent Van Gogh (Lettres à son frère Théo, Grasset, 1937) 
106

nul, ne parvient pour nous à quelque justification que si ces amateurs possèdent des moyens suffisants, nous voulons dire sur le plan financier, pour que leur plaisir s'impose à nous comme une action respectable. Autrement, ce n'est qu'enfantillage et insulte aux intérêts légitimes de l'humanité souffrante dite aussi ordinaire. Nous avons hâte d'entendre Bartolomeo sur ce point.

Quand, la veille de son achat, il tentait de prouver que le Messerschmitt PK 33 de 1917, dont on lui proposait un modèle assez rare, avait failli, par son avance technologique, donner la victoire à l'Allemagne, je m'étais élevé en faux, lui rétorquant l'épisode de Milbertshofen: trois cents appareils cloués au sol par le même défaut technique: une faiblesse du chevalet qui, sur cet appareil, tenait les ailes. J'ai encore réfléchi au sujet de notre conversation, et involontairement j'ai songé aux paroles "nous sommes aujourd'hui ce que nous étions hier". Cela ne signifie

Aujourd'hui nous sommes bien décidés à le forcer à s'expliquer là-dessus. Il ne pourra se défiler. Il lui faudra bien se justifier. Dire ce qu'il pense vraiment des collectionneurs. Des investisseurs. De tous ceux qui prennent les toiles pour du numéraire. Ce n'est pas que nous soyons à cheval sur les principes, ni même que nous pensions que l'art n'a pas de prix mais les faiblesses coupables qu'ont, de nos jours, les appareils idéologiques d'État, comme on dit, à l'endroit de forces que leur anonymat et leur caractère d'imprévisibilité font un peu vite qualifier

pas que l'on doive marquer notre passage sur cette terre par une sorte de fidélité à nos goûts d'enfants, à notre passion, par exemple, pour les aéroplanes teutons, les machines volantes germaniques ou les bi-plans prussiens, lesquels au demeurant n'étaient pas aussi efficaces, du moins à cette époque, qu'il le prétendait avec véhémence, il n'y avait qu'à voir ce qui était arrivé à toutes ces escadrilles, à Milbertshofen, où il avait suffi du goût immodéré qu'éprouvait l'ingénieur chargé de leur entretien pour la peinture des primitifs flamands d'une part et le chocolat

d'économiques, ces faiblesses, disons-nous, encouragent les jeunes et le grand public à ne voir dans toute entreprise humaine que son efficacité immédiate et surtout indubitable, le monnayage des dites n'étant dès lors que la sanction sociale apposée indiscutablement ou refusée irrémédiablement à ce qui pourtant, comme l'art, échappe à une telle sanction, du moins quand celle-ci prend cette forme. Que diable peut-il bien faire? Voilà bientôt trente 
minutes... Et le café qui refroidit! Si c'est ainsi qu'il s'est occupé du Van Goff, pas étonnant qu'il n'ait pas eu plus de

d'autre part, pour que toute l'armada soit immobilisée à découvert, à la merci des bombes françaises et anglaises, si bien qu'en l'espace de quelques minutes les trois cents Messerschmitt PK se sont pour ainsi dire volatilisés, partis en fumée tandis que l'ingénieur se repaîssait de celui d'un chocolat des plus onctueux en pensant à l'œuvre de Van der Weyden qu'il venait de voir dans la ville occupée de Cambrai, célèbre pour ses bêtises, sortes de sucres d'orge multicolores, celle de l'ingénieur ayant précisément consisté à oublier de raffermir les chevalets dont il s'était aperçu

succès avec lui que le mois précédent avec la grande et brutale eau-forte, d'après Rembrandt, intitulée, croyons-nous, Le jardin des Olives et attribuée, nous semble-t-il maintenant, à Carlo Dolci. Pour quelle raison une maison aussi sérieuse garde-t-elle à son service un individu aussi fantasque et nonchalant, c'est ce qui décidément nous étonnera toujours. Il n'en est pas à sa première bourde, le Bartolomeo, et lui confier une ouvre de Van Goff, surtout s'il s'agit de L'esprit de Saint Louis, une ouvre qui n'avait jusqu'ici traversé l'Atlantique qu'une fois,

la veille qu'ils demandaient quelque solidification, se contentant sur le coup de commander que les trois cents appareils soient sortis de leurs hangars et simplement recouverts de toiles tenues par des cordages un peu lâches, de sorte qu'il serait facile, quand l'ingénieur en question y serait disposé, de passer de l'un à l'autre, sur la piste, avec une équipe d'ouvriers, et d'effectuer les réparations exigées, tandis que derrière eux un peintre ou deux marqueraient les bâches de ceux qui viendraient d'être réparés d'une inscription du genre "gemacht " ou d'un

c'est faire preuve pour le moins d'imprévoyance. Bien sûr, c'est une autorité sur Guido Reni et sur Van Houtt, son disciple hollandais, mais nous imaginons qu'il ne doit pas s'en vendre tous les jours des tableaux de cette école qui ne savait faire que des madonnes. D'autant moins que l'heure est aux paysages. Oui, nous savons bien que Van Goff lui-même disait: "Si je fais des paysages, il y aura toujours là-dedans trace de figures ", mais tout de même, son Esprit de Saint Louis n'est pas une métaphore, c'est bel et bien l'engin lui-même échoué dans une verdure criarde,

trait plus abstrait, un carré de couleur ou un rectangle, bleu, rouge, jaune, comme dans l'œuvre de ce peintre allemand dont 
108

le nom m'échappe, celui qui a fait Le voyage de Saint Ubald... C'est trop bête!, je ne connais que lui!, enfin, j'imagine que ça me reviendra, toujours est-il que tandis qu'à Cambrai l'ingénieur se livrait aux délices combinés du cacao et de Rogier de la Pasture, les trois cents ouailles volantes dont il avait la garde disparaissaient purement et simplement dans le fracas des bombes et l'incessant ballet des pinceaux lumineux sur lesquels de

savants entrelacs de branches et de feuilles où disparaît presque l'appareil, réduit par l'habileté du peintre à une vague tâche jaunâtre dont pourtant nul, la regardant, ne douterait un seul instant qu'elle représente autre chose que cet objet dont la renommée a fait le tour du monde. Nous lui donnerons encore dix minutes, après quoi, sans laisser le moindre message à son intention à la secrétaire ni même quelque papier plié sur la porte, remettant sans un soupir notre chéquier dans notre poche, un sourire aux lèvres mais la rage au cœur, nous nous éclipserons.

besogneux artilleurs (ou dit-on "servants"?) tentaient de régler leur tir à la recherche d'invisibles assaillants bourdonnant dans la nuit. Théo pouvait bien prétendre que n'eût été l'incurie de l'ingénieur en question l'avion dont il convoitait le modèle réduit aurait aisément surmonté ce très léger problème technique, pris l'air, ce jour-là, les trois cents appareils comme un seul, liquidé les escadrilles ennemies, donné la victoire à l'Allemagne, ouvert la porte, seul dans ce wagon, lu sur le mur l'inscription "Van Horne" où j'attendais "Berri ", pesté, sacré

Juré, c'est juré, mais un peu tard sans doute, on ne nous y reprendra plus. Ramasser nos affaires, calmement, sans nous presser, aller à la patère, décrocher notre manteau, le mettre, quand finalement la porte s'ouvre et nous le voyons entrer. Nous lui sourions et

sauté sur le quai, et dire que j'espérais, pris un taxi, lui vendre, l'ascenseur, le tableau, précipité dans le couloir, de Van Goff, essoufflé, hors de moi quand enfin je parviens à la porte de son bureau, l'ouvre et me retrouve devant ce fat qui parle de luimême au pluriel. Plus qu'à s'excuser, s'avancer encore et

Nous nous serrons la main. 